\title{
The Co-Points of Rays are Cut Points of Upper Level Sets for Busemann Functions
}

\author{
Sorin V. SABAU
}

School of Science, Department of Mathematics, Tokai University, Sapporo 005-8600, Japan

E-mail:ssorin@tokai.ac.jp

Received August 07, 2015, in final form April 06, 2016; Published online April 13, 2016

http://dx.doi.org/10.3842/SIGMA.2016.036

\begin{abstract}
We show that the co-rays to a ray in a complete non-compact Finsler manifold contain geodesic segments to upper level sets of Busemann functions. Moreover, we characterise the co-point set to a ray as the cut locus of such level sets. The structure theorem of the co-point set on a surface, namely that is a local tree, and other properties follow immediately from the known results about the cut locus. We point out that some of our findings, in special the relation of co-point set to the upper lever sets, are new even for Riemannian manifolds.
\end{abstract}

Key words: Finsler manifolds; ray; co-ray (asymptotic ray); cut locus; co-points; distance function; Busemann function

2010 Mathematics Subject Classification: 53C60; 53C22

\section{Introduction}

Roughly speaking, a Busemann function is a function that measures the distance to a point at infinity on a complete boundaryless non-compact Riemannian or Finsler manifold. Originally introduced by $\mathrm{H}$. Busemann for constructing a theory of parallels for straight lines (see $[2,4,5$, 10]), the function plays a fundamental role in the study of complete non-compact Riemannian or Finsler manifolds (see [8, 10, 11], etc).

In the present paper, we study the differentiability of the Busemann function in terms of co-rays and co-points to a ray in the general case of a forward complete non-compact Finsler manifold. We show that the notions of geodesic segments to a closed subset and the cut locus of such sets can be extremely useful in the study of co-rays and co-points to a ray, that is points where Busemann function is not differentiable.

The originality of our research is two folded. Firstly, the detailed study of Busemann functions, co-rays and co-points on Finsler manifolds is new. Secondly, in the special case of Riemannian manifolds, our main Theorems 1.1 and 1.2, first statement, are new and they lead to new elementary proofs of other results already known.

Let $(M, F)$ be a forward complete boundaryless Finsler manifold. A unit speed globally minimising geodesic $\gamma:[0, \infty) \rightarrow M$ is called a (forward) ray. A ray $\gamma$ is called maximal if it is not a proper sub-ray of another ray, i.e., for any $\varepsilon>0$ its extension to $[-\varepsilon, \infty)$ is not a ray anymore. Moreover, let us assume that $(M, F)$ is bi-complete, i.e., forward and backward complete. A Finslerian unit speed globally minimising geodesic $\gamma: \mathbb{R} \rightarrow M$ is called a straight line. We point out that, even though for defining rays and straight lines we not need any completeness hypothesis, without completeness, introducing rays and straight lines would be meaningless.

Let $\gamma:[0, \infty) \rightarrow M$ be a given forward ray and let $x$ be a point on a non-compact forward complete Finsler manifold $(M, F)$. Then, a forward ray $\sigma:[0, \infty) \rightarrow M$ is called a forward co-ray (or a forward asymptotic ray) to $\gamma$ if there exists a sequence of minimal geodesics $\left\{\sigma_{j}\right\}$ 
from $q_{j}:=\sigma_{j}(0)$ to $\sigma_{j}\left(l_{j}\right):=\gamma\left(t_{j}\right)$, for some divergent sequence of numbers $\left\{t_{j}\right\}$, such that $\lim _{j \rightarrow \infty} q_{j}=\sigma(0)$ and $\dot{\sigma}(0)=\lim _{j \rightarrow \infty} \dot{\sigma}_{j}(0)$.

A co-ray to $\gamma$ is called maximal if for any $\varepsilon>0$ its extension to $[-\varepsilon, \infty)$ is not an co-ray to $\gamma$ anymore. The origin points of maximal co-rays of $\gamma$ are called the co-points to $\gamma$ (a slightly stronger definition can be found in [8]).

Similarly, one can define asymptotic straight lines. If $\gamma: \mathbb{R} \rightarrow M$ is a straight line in a bicomplete Finsler manifold, then the straight line $\sigma: \mathbb{R} \rightarrow M$ is an asymptotic straight line to $\gamma$ if $\left.\sigma\right|_{[0, \infty)}$ is asymptotic ray to $\left.\gamma\right|_{[0, \infty)}$, and $\left.\bar{\sigma}\right|_{[0, \infty)}$ is asymptotic ray to $\left.\bar{\gamma}\right|_{[0, \infty)}$ with respect to the reverse Finsler metric $\bar{F}(x, y):=F(x,-y)$, where $\bar{\sigma}(t):=\sigma(-t)$ and $\bar{\gamma}(t):=\gamma(-t), t \in[0, \infty)$ are the reverse rays of $\sigma$ and $\gamma$, respectively (see [8] for details). This definition makes sense because if $\sigma$ is a geodesic for $F$, then the reverse curve $\bar{\sigma}(t)$ is geodesic for $\bar{F}$.

If $\gamma$ is a forward ray in the forward complete boundaryless non-compact Finsler manifold $(M, F)$, then the Busemann function is defined by $x \in M \mapsto \mathbf{b}_{\gamma}(x):=\lim _{t \rightarrow \infty}\{t-d(x, \gamma(t))\}$, where $d$ is the Finsler distance function (see Section 2 for details).

Let us recall from [12] some notions that will be useful later.

Let $N \subset M$ be a closed subset of $M$. For a point $p \in M \backslash N$, a unit speed geodesic segment $\alpha:[0, a] \rightarrow M$ from $p=\alpha(0)$ is called a forward $N$-segment if $d(\alpha(t), N)=a-t$ holds on $[0, a]$, where $d(x, N):=\inf \{d(x, q): q \in M\}$ for any $x \in M$. The existence of $N$-segments from any $p \in M \backslash N$ follows from the fact that $N$ is closed and the forward completeness hypothesis. If a unit speed (nonconstant) geodesic segment $\alpha:[0, a] \rightarrow M$ is maximal as an $N$-segment, then the point $p=\alpha(0)$ is called a cut point of $N$ along the $N$-segment $\alpha$, i.e., any geodesic extension $\tilde{\alpha}:[-\varepsilon, a] \rightarrow M, \varepsilon>0,\left.\tilde{\alpha}\right|_{[0, a]}=\left.\alpha\right|_{[0, a]}$ of $\alpha$ is not a forward $N$-segment anymore. The cut locus of $N$, denoted hereafter $\mathcal{C}_{N}$, is the set of all cut points of $N$ along all nonconstant $N$-segments. Observe that $\mathcal{C}_{N} \cap N=\varnothing$. If a point $p \in M \backslash N$ admits two $N$-segments of equal length, then $p$ is a cut point of $N$. Therefore, any interior point of $N$-segment is not a cut point of $N$.

We point out that in [12], for a closed subset $N$ of a backward complete Finsler manifold $(M, F)$, a backward $N$-segment is defined analogously. The notions of forward and backward $N$-segments to a closed subset $N$ are equivalent. Indeed, if we consider the reverse Finsler metric $\widetilde{F}$ on the manifold $M$ given by $\widetilde{F}(x, y):=F(x,-y)$ for each $(x, y) \in T M$, a backward $N$-segment on $(M, F)$ is a forward $N$-segment on $(M, \widetilde{F})$.

Notice that, since we consider only boundaryless manifolds, any geodesic segment on a compact interval admits forward and backward local geodesic extensions even if the manifold $M$ is not forward nor backward complete. For more basics on Finsler manifolds see [1] or [9].

Here are the main results of our paper.

Theorem 1.1. Let $(M, F)$ be a forward complete boundaryless Finsler manifold and let $\alpha:[0, a]$ $\rightarrow M$ be a unit-speed geodesic. The following three statements are equivalent.

1. $\alpha$ is a subarc of a co-ray to $\gamma$.

2. $\alpha$ satisfies

$$
\mathbf{b}_{\gamma}(\alpha(s))=s+\mathbf{b}_{\gamma}(\alpha(0))
$$

for all $s \in[0, a]$.

3. $\alpha$ is a forward $N_{\gamma}^{b}$-segment, where $N_{\gamma}^{b}:=\mathbf{b}_{\gamma}^{-1}[b, \infty)$ and $b=\mathbf{b}_{\gamma}(\alpha(a))$.

From here the relation between co-points to a forward ray and the cut points of a level set of Busemann function naturally follows.

Theorem 1.2. Let $(M, F)$ be a forward complete boundaryless Finsler manifold, and $\gamma$ a ray in $M$. 
1. For every $b \in \mathbb{R}$, the set of co-points of $\gamma$ in the sub-level $\mathbf{b}_{\gamma}^{-1}(-\infty, b)$ is exactly the cut locus of the subset $N_{\gamma}^{b}$, i.e., $\mathcal{C}_{\gamma} \cap \mathbf{b}_{\gamma}^{-1}(-\infty, b)=\mathcal{C}_{N_{\gamma}^{b}}$. Moreover, $\mathcal{C}_{N_{\gamma}^{b}} \subset \mathcal{C}_{N_{\gamma}^{c}}$, for any $b<c$.

2. The Busemann function $\mathbf{b}_{\gamma}$ is differentiable at a point $x$ of $M$ if and only if $x$ admits a unique co-ray $\sigma$ to $\gamma$ emanating from $x=\sigma(0)$. In this case $\nabla \mathbf{b}_{\gamma}(x)=\dot{\sigma}(0)$.

Loosely speaking, $\mathcal{C}_{\gamma}=\bigcup_{b} \mathcal{C}_{N_{\gamma}^{b}}$, where $\mathcal{C}_{N_{\gamma}^{b}}$ denotes the cut locus of $N_{\gamma}^{b}$. Here "loosely" means that it is possible that the local part of $\mathcal{C}_{N_{\gamma}^{b}}$ near a fixed point $x$ keeps changing and never stabilises as $b$ goes to $+\infty$.

These two main theorems make possible to apply known results about the $N$-segments and cut points of a closed subset of $(M, F)$ to the study of co-rays and co-points, allowing the use of our previous results from [12].

Seen in this light, the proof of the structure theorem for the co-point set on a Finsler surface, namely that is a local tree, becomes trivial. It is also clear that the topology of $\left(\mathcal{C}_{\gamma}, \delta\right)$, with the induced metric, coincides with the topology of the Finsler surface, as well as that $\left(\mathcal{C}_{\gamma}, \delta\right)$ is forward complete (see Theorem 2.13). Other results are also straightforward from [12] (see Theorem 2.14).

Section 3 contains some consequences of the characterisation of the Busemann function's differentiability given above. Here we study the conditions for the set $\mathbf{b}_{\gamma}^{-1}(-\infty, c]$ to be compact (Theorem 3.1), and for $\mathbf{b}_{\gamma}$ to be an exhaustion (Corollary 3.3). If the co-point set $\mathcal{C}_{\gamma}$ contains an isolated point, then some important consequences are proved in Theorem 3.6.

\section{Busemann functions}

Let $(M, F)$ be a forward complete boundaryless non-compact Finsler manifold (see [1,9] for details on the completeness of Finsler manifolds). In Riemannian geometry, the forward and backward completeness are equivalent, hence the words "forward" and "backward" are superfluous, but in Finsler geometry these are not equivalent anymore.

Definition 2.1. If $\gamma:[0, \infty) \rightarrow M$ is a ray in a forward complete boundaryless non-compact Finsler manifold $(M, F)$, then the function

$$
\mathbf{b}_{\gamma}: M \rightarrow \mathbb{R}, \quad \mathbf{b}_{\gamma}(x):=\lim _{t \rightarrow \infty}\{t-d(x, \gamma(t))\}
$$

is called the Busemann function with respect to $\gamma$, where $d$ is the Finsler distance function.

The Busemann function for Finsler manifolds was introduced and partially studied by Egloff [3] and more recently by [8].

\section{Remark 2.2.}

1. The limit in (2.1) always exists because the function $t \mapsto t-d(x, \gamma(t))$ is monotone nondecreasing and bounded above by $d(\gamma(0), x)$.

2. Obviously $\mathbf{b}_{\gamma}(\gamma(t))=t$, for all $t \geq 0$. Moreover, if $\gamma_{0}$ is a sub-ray of the ray $\gamma$, then $\mathbf{b}_{\gamma_{0}}(x)=\mathbf{b}_{\gamma}(x)-t_{0}$ for any point $x \in M$, where $t_{0} \geq 0$ is the parameter value on $\gamma$ such that $\gamma_{0}(0)=\gamma\left(t_{0}\right)$.

It follows that a point $x$ of $M$ is an element of $\mathbf{b}_{\gamma}^{-1}(a, \infty)$, for some real number $a$, if and only if $t-d(x, \gamma(t))>a$ for some $t>0$, and hence we get

Lemma 2.3. For each $a \in \mathbb{R}, \mathbf{b}_{\gamma}^{-1}(a, \infty)=\bigcup_{t>0} B_{t}^{-}(\gamma(t+a))$ holds, where $B_{t}^{-}(\gamma(t+a)):=$ $\{x \in M \mid d(x, \gamma(t+a))<t\}$ denotes the backward open ball centred at $\gamma(t+a)$ of radius $t$. In particular $\mathbf{b}_{\gamma}^{-1}(a, \infty)$ is arcwise connected for each $a \geq 0$. 
The triangle inequality implies

Lemma 2.4. The function $\mathbf{b}_{\gamma}$ is locally Lipschitz, i.e.,

$$
-d(x, y) \leq \mathbf{b}_{\gamma}(x)-\mathbf{b}_{\gamma}(y) \leq d(y, x)
$$

for any two points $x, y \in M$.

The differentiability of Busemann function is fundamental for the study of co-rays. Some results are already known (see for instance [4]). Let us denote by $\nabla f(x)$ the Finslerian gradient of a smooth function $f: M \rightarrow \mathbb{R}$ (see [8] or [9, p. 41]).

Theorem $2.5([8])$. Let $\gamma$ be a forward ray in a non-compact forward complete Finsler manifold $(M, F)$.

1. For any $x \in M$, there exists at least one co-ray $\sigma$ of $\gamma$ such that $\sigma(0)=x$.

2. If the geodesic ray $\sigma$ is a co-ray to $\gamma$, then $\mathbf{b}_{\gamma}(\sigma(s))=s+\mathbf{b}_{\gamma}(\sigma(0)), \forall s \geq 0$.

3. If $\mathbf{b}_{\gamma}$ is differentiable at a point $x \in M$, then $\sigma(s):=\exp _{x}\left(s \nabla \mathbf{b}_{\gamma}(x)\right)$ is the unique co-ray to $\gamma$ emanating from $x$, where $\nabla \mathbf{b}_{\gamma}(x)$ is the Finslerian gradient of $\mathbf{b}_{\gamma}$ at $x$.

Remark 2.6. The converse of statement 2 in Theorem 2.5 is actually contained in our Theorem $1.1,2 \Rightarrow 1$.

For any closed subset $N$ of $M$, we have defined $N$-segments in Introduction. From now on, any $N_{\gamma}^{b}$-segment will mean forward $N_{\gamma}^{b}$-segment, where $N_{\gamma}^{b}:=\mathbf{b}_{\gamma}^{-1}[b, \infty)$.

Proof of Theorem 1.1. $1 \Rightarrow 2$. Suppose that the property 1 holds. Then, statement 2 follows immediately from Theorem $2.5(2)$.

$2 \Rightarrow 3$. Choose any $s \in[0, a]$ and any $x \in \mathbf{b}_{\gamma}^{-1}[b, \infty)$, where $b:=\mathbf{b}_{\gamma}(\alpha(a))$. By definition we have $\mathbf{b}_{\gamma}(x) \geq b=\mathbf{b}_{\gamma}(\alpha(a))$, and from Lemma 2.4 it follows

$$
\mathbf{b}_{\gamma}(\alpha(a))-\mathbf{b}_{\gamma}(\alpha(s)) \leq \mathbf{b}_{\gamma}(x)-\mathbf{b}_{\gamma}(\alpha(s)) \leq d(\alpha(s), x) .
$$

On the other hand, the relation (1.1) implies

$$
d(\alpha(s), \alpha(a)) \leq a-s=\mathbf{b}_{\gamma}(\alpha(a))-\mathbf{b}_{\gamma}(\alpha(s)) .
$$

From relations (2.2) and (2.3) it results $d(\alpha(s), \alpha(a))=d\left(\alpha(s), N_{\gamma}^{b}\right)$ for any $s \in[0, a]$, and since the point $x$ is arbitrarily chosen from $N_{\gamma}^{b}$ we obtain that $\alpha$ is an $N_{\gamma}^{b}$-segment.

$3 \Rightarrow 1$. Choose any sufficiently small $\varepsilon>0$. Let $\sigma_{\varepsilon}:[\varepsilon, \infty) \rightarrow M$ denote a co-ray to $\gamma$ emanating from $\alpha(\varepsilon),\left.\alpha\right|_{(\varepsilon, \infty)} \neq\left.\sigma_{\varepsilon}\right|_{(\varepsilon, \infty)}$.

Since $\sigma_{\varepsilon}$ satisfies (1.1) for all $s \geq \varepsilon,\left.\sigma\right|_{[\varepsilon, a]}$ is also an $N_{\gamma}^{b}$-segment emanating from $\alpha(\varepsilon)$. Thus, the two geodesic segments $\left.\alpha\right|_{[\varepsilon, a]}$ and $\left.\sigma_{\varepsilon}\right|_{[\varepsilon, a]}$ must coincide, since $\alpha(\varepsilon)$ is an interior point of $\alpha$ and interior points of $N$-segments have a unique $N$-segment. Therefore, $\alpha$ is a subarc of the co-ray $\lim _{\varepsilon \rightarrow 0} \sigma_{\varepsilon}$.

By Theorem 1.1 we get

Corollary 2.7. If a unit speed geodesic $\sigma:[0, a] \rightarrow M$ satisfies relation (1.1), for all $s \in[0, a]$, then $\sigma$ is a co-ray to $\gamma$.

Corollary 2.8. For each $a \in \mathbb{R}$ such that $\mathbf{b}_{\gamma}^{-1}(a) \neq \varnothing$, we have

$$
d\left(x, N_{\gamma}^{a}\right)=a-\mathbf{b}_{\gamma}(x), \quad \forall x \in \mathbf{b}_{\gamma}^{-1}(-\infty, a] .
$$

Hence, $\mathbf{b}_{\gamma}$ is differentiable at a point $x$ if and only if for each real number $a>\mathbf{b}_{\gamma}(x)$ the distance function $d\left(\cdot, N_{\gamma}^{a}\right)$ is differentiable at $x$. 
Proof. Choose any $x \in \mathbf{b}_{\gamma}^{-1}(-\infty, a]$, and denote by $\sigma:[0, \infty) \rightarrow M$ a co-ray to $\gamma$ emanating from $x=\sigma(0)$. Since $\left.\sigma\right|_{\left[0, a-\mathbf{b}_{\gamma}(x)\right]}$ is an $N_{\gamma}^{a}$-segment and noticing that $\sigma\left(a-\mathbf{b}_{\gamma}(x)\right) \in \mathbf{b}_{\gamma}^{-1}(a)$, we obtain $d\left(x, N_{\gamma}^{a}\right)=d\left(\sigma(0), \sigma\left(a-\mathbf{b}_{\gamma}(x)\right)\right)=a-\mathbf{b}_{\gamma}(x)$.

Proof of Theorem 1.2. Let $x \in M$ be any co-point of $\gamma$. Choose $b>0$ such that $b>\mathbf{b}_{\gamma}(x)$. Then, from Theorem 1.1 it follows that, for any co-ray $\sigma:[0, \infty) \rightarrow M$ of $\gamma$ from $x$, we have

- the relation $\mathbf{b}_{\gamma}(\sigma(s))=s+\mathbf{b}_{\gamma}(\sigma(0))=s+\mathbf{b}_{\gamma}(x)$ holds good for any $s \geq 0$. Hence, for our chosen $b>0$, there always exists $a>0$ such that $b=\mathbf{b}_{\gamma}(\sigma(a))=a+\mathbf{b}_{\gamma}(x)$;

- the geodesic segment $\left.\sigma\right|_{[0, a]}$ is a maximal $N_{\gamma}^{b}$-segment.

It results that $x \in \mathcal{C}_{N_{\gamma}^{b}}$.

Conversely, we choose any point $x \in \cup_{b>0} \mathcal{C}_{N_{\gamma}^{b}}$. It follows that $x$ is a cut point of $N_{\gamma}^{b}$, for some $b>0$. Let $\sigma:[0, a] \rightarrow M$ be an $N_{\gamma}^{b}$-segment from $x=\sigma(0)$, where $b=\mathbf{b}_{\gamma}(\sigma(a))=a+\mathbf{b}_{\gamma}(x)$. Theorem 1.1 implies that there exists a maximal co-ray $\widetilde{\sigma}:[0, \infty) \rightarrow M$ of $\gamma$ such that $\left.\widetilde{\sigma}\right|_{[0, a]}=$ $\left.\sigma\right|_{[0, a]}$. This means that $x=\widetilde{\sigma}(0) \in \mathcal{C}_{\gamma}$.

We will prove now that $\mathcal{C}_{N_{\gamma}^{b}} \subset \mathcal{C}_{N_{\gamma}^{c}}$, for any $b<c$. Indeed, let us consider any point $x \in \mathcal{C}_{N_{\gamma}^{b}}$, and let $\sigma:[0, a] \rightarrow M$ be an $N_{\gamma}^{b}$-segment emanating from $x=\sigma(0)$, i.e., $b=\mathbf{b}_{\gamma}(\sigma(a))=a+\mathbf{b}_{\gamma}(x)$.

Notice that any short backward geodesic extension $\tilde{\sigma}:[-\varepsilon, a] \rightarrow M$ of $\sigma$, where $\varepsilon>0$, cannot be an $N_{\gamma}^{b}$-segment, due to the fact that $x \in \mathcal{C}_{N_{\gamma}^{b}}$.

On the other hand, by Theorem $1.1,\left.\sigma\right|_{[0, a]}$ is a subarc of a maximal co-ray $\widetilde{\sigma}:[0, \infty) \rightarrow M$ of $\gamma$. Taking into account that $a+\mathbf{b}_{\gamma}(x)=b<c$, that is, there exists $\widetilde{a}>0$ such that $a<\widetilde{a}:=c-\mathbf{b}_{\gamma}(x)$, then again from Theorem 1.1 it results that the subarc $\left.\widetilde{\sigma}\right|_{[0, \widetilde{a}]}$ is a maximal

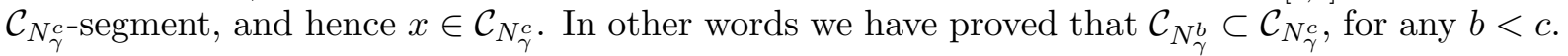

2. Follows easily from Theorem A in [12], Corollary 2.8 and Theorem 1.1.

Corollary 2.9. If $x \in M$ is an interior point of a co-ray $\sigma$ of $\gamma$, then $\mathbf{b}_{\gamma}$ is differentiable at $x$.

Proof. Choose any point $\sigma\left(t_{0}\right), t_{0}>0$. By Theorem 1.1, the subray $\left.\sigma\right|_{\left[t_{0}, \infty\right)}$ is a unique co-ray to $\gamma$ emanating from $\sigma\left(t_{0}\right)$. Thus, Theorem 1.2, statement 2 shows that $\mathbf{b}_{\gamma}$ is differentiable at $\sigma\left(t_{0}\right)$.

Let us denote by $\mathcal{C}_{\gamma}$ the co-point set of the ray $\gamma$, that is the origin points of the maximal co-rays to $\gamma$.

Remark 2.10. From the definition of co-points it follows that if $p \in \mathcal{C}_{\gamma}$, then there exists a maximal co-ray of $\gamma$ emanating from $p$. Equivalently, any co-ray emanating from $p \in \mathcal{C}_{\gamma}$ is maximal.

By Proposition 2.5 in [12] and our Theorem 1.1 we obtain the following.

Corollary 2.11. Let $(M, F)$ be a forward complete boundaryless Finsler manifold, $\gamma$ a forward ray in $M$ and $\mathcal{C}_{\gamma}$ the co-point set of $\gamma$.

Then, the subset

$$
\mathcal{C}_{\gamma}^{(2)}:=\left\{p \in \mathcal{C}_{\gamma}: \text { there exist at least two maximal co-rays from } p \text { to } \gamma\right\} \subset \mathcal{C}_{\gamma}
$$

is dense in $\mathcal{C}_{\gamma}$.

Remark 2.12. Let $\mathcal{N D}\left(\mathbf{b}_{\gamma}\right) \subset M$ be the set of non-differentiable points of the Busemann function $\mathbf{b}_{\gamma}$. Then, from Corollaries 2.9 and 2.11 it follows $\mathcal{C}_{\gamma}^{(2)}=\mathcal{N D}\left(\mathbf{b}_{\gamma}\right) \subset \mathcal{C}_{\gamma} \subset \overline{\mathcal{N} \mathcal{D}\left(\mathbf{b}_{\gamma}\right)}$.

In the special case when $\mathcal{C}_{\gamma}$ is closed set in $M$, we have $\mathcal{N D}\left(\mathbf{b}_{\gamma}\right) \subset \mathcal{C}_{\gamma}=\overline{\mathcal{N D}\left(\mathbf{b}_{\gamma}\right)}$. This is not true in general (see Remark 3.10). 
In the two dimensional case, the structure theorems of the cut locus from [12] can be easily extendend to the structure of $\mathcal{C}_{\gamma}$. We recall that an injective continuous map from the open interval $(0,1)$ (or closed interval $[0,1]$ ) of $\mathbb{R}$ and from a circle $\mathbb{S}^{1}$ into $M$ is called a Jordan arc and a Jordan curve, respectively.

A topological space $T$ is called a tree if any two points in $T$ can be joined by a unique Jordan arc in $T$. Likewise, a topological space $C$ is called a local tree if for every point $x \in C$ and for any neighborhood $U$ of $x$, there exists a neighborhood $V \subset U$ of $x$ such that $V$ is a tree.

A continuous curve $c:[a, b] \rightarrow M$ is called rectifiable if its length

$$
l(c):=\sup \left\{\sum_{i=1}^{k} d\left(c\left(t_{i-1}\right), c\left(t_{i}\right)\right) \mid a=: t_{0}<t_{1}<\cdots<t_{k-1}<t_{k}:=b\right\} .
$$

is finite.

By Theorem 1.2 and Theorem B in [12] we obtain (compare with [6])

Theorem 2.13. Let $\gamma$ be a ray in a forward complete boundaryless 2-dimensional Finsler manifold $(M, F)$. Then, the of co-point set $\mathcal{C}_{\gamma}$ of $\gamma$ satisfies the following three properties.

1. The set $\mathcal{C}_{\gamma}$ is a local tree and any two co-points on the same connected component of $\mathcal{C}_{\gamma}$ can be joined by a rectifiable curve in $\mathcal{C}_{\gamma}$.

2. The topology of $\mathcal{C}_{\gamma}$ induced from the intrinsic metric $\delta$ (see definition below) coincides with the induced topology of $\mathcal{C}_{\gamma}$ from $(M, F)$.

3. The metric space $\mathcal{C}_{\gamma}$ with the intrinsic metric $\delta$ is forward complete.

Indeed, by the first statement, any two co-points $q_{1}, q_{2} \in \mathcal{C}_{\gamma}$ can be joined by a rectifiable arc in $\mathcal{C}_{\gamma}$ if $q_{1}$ and $q_{2}$ are in the same connected component. Therefore, the intrinsic metric $\delta$ on $\mathcal{C}_{\gamma}$ defined as

$$
\delta\left(q_{1}, q_{2}\right):=\left\{\begin{array}{l}
\inf \left\{l(c) \mid c \text { is a rectifiable arc in } \mathcal{C}_{\gamma} \text { joining } q_{1} \text { and } q_{2}\right\} \\
\quad \text { if } q_{1}, q_{2} \in \mathcal{C}_{\gamma} \text { are in the same connected component } \\
+\infty, \quad \text { otherwise }
\end{array}\right.
$$

is well defined.

By Theorem 1.2 and Theorem $\mathrm{C}$ in [12] we have

Theorem 2.14. Let $\gamma$ be a ray in a forward complete boundaryless 2-dimensional Finsler manifold $(M, F)$. Then, there exists a set $\mathcal{E} \subset[0, \infty)$ of measure zero with the following properties:

1. For each $t \in(0, \infty) \backslash \mathcal{E}$, the set $\mathbf{b}_{\gamma}^{-1}(t)$ consists of locally finitely many mutually disjoint arcs. In particular, if $\mathbf{b}_{\gamma}^{-1}(a)$, is compact for some $a>t$, then $\mathbf{b}_{\gamma}^{-1}(t)$ consists of finitely many mutually disjoint circles.

2. For each $t \in(0, \infty) \backslash \mathcal{E}$, any point $q \in \mathbf{b}_{\gamma}^{-1}(t)$ admits at most two maximal co-rays.

Here locally finitely many means that for $x \in \mathbf{b}_{\gamma}(t)$, and any forward (or backward) ball $\mathcal{B}^{+}(x, r):=\{p \in M: d(x, p)<r\}$, the set $\mathcal{B}^{+}(x, r) \cap \mathbf{b}_{\gamma}(t)$ contains only finitely many arcs.

\section{Implications of the differentiability of $b_{\gamma}$}

Here are some results that follow from the previous section (compare with [4]).

In [4] it is proved for $G$-spaces that if the co-point set $\mathcal{C}_{\gamma} \neq \varnothing$ is compact, then $\mathbf{b}_{\gamma}$ is an exhaustion function. We will give a more general result. 
Theorem 3.1. Let $(M, F)$ be a forward complete non-compact boundaryless Finsler manifold and $\gamma$ a ray in $M$.

If for some given $c \geq \inf \mathbf{b}_{\gamma}(M)$, the set $\mathcal{C}_{\gamma} \cap \mathbf{b}_{\gamma}^{-1}(-\infty, c]$ is compact and non-empty, then $\mathbf{b}_{\gamma}^{-1}(-\infty, c] \neq \varnothing$ is compact.

Proof. For the number $c \geq \inf \mathbf{b}_{\gamma}(M)$ given, we define the set

$S_{c}:=\left\{q \in \mathbf{b}_{\gamma}^{-1}(c) \mid q\right.$ belongs to some co-ray to $\gamma$ emanating from a point in $\left.\mathcal{C}_{\gamma}\right\}$.

For later use we also define

$$
\begin{aligned}
\widetilde{M}:=\left\{x \in M \mid \text { there is a maximal co-ray } \sigma_{x}:(a, \infty) \rightarrow M, \text { passing through } x,\right. \\
\\
\quad \text { for some } a \in[-\infty, \infty)\} .
\end{aligned}
$$

Remark that if $F$ is bi-complete, then $a=-\infty$ always in the definition of $\widetilde{M}$, but since we assume only forward completeness here, a finite value for $a$ might happen.

We will divide the proof in two steps.

Step 1. Firstly, we prove that

$$
S_{c}=\mathbf{b}_{\gamma}^{-1}(c) \text {. }
$$

In the case $c<\inf \mathbf{b}_{\gamma}(M)$, we prove this by showing the followings

(i) the set $S_{c}$ is non-empty,

(ii) $S_{c}$ is open in $\mathbf{b}_{\gamma}^{-1}(c)$,

(iii) $\widetilde{M}$ is closed in $M$,

(iv) $\widetilde{M}$ is open in $M$.

Proof of (i). Firstly, we show that $S_{c} \neq \varnothing$. Indeed, taking into account the hypothesis, we can consider a point $p \in \mathcal{C}_{\gamma} \cap \mathbf{b}_{\gamma}^{-1}(-\infty, c]$. If $\mathbf{b}_{\gamma}(p)=c$ then $p \in S_{c}$ and there is nothing to prove.

We can therefore assume $\mathbf{b}_{\gamma}(p)<c$, that is there exists $l>0$ such that $\mathbf{b}_{\gamma}(p)=c-l$.

Since $p \in \mathcal{C}_{\gamma}$, we consider the maximal co-ray $\sigma:[0, \infty) \rightarrow M$ from $p$ to $\gamma$ and let $q$ be the point on $\sigma$ such that $d(p, q)=l$. Then $\mathbf{b}_{\gamma}(\sigma(s))=s+\mathbf{b}_{\gamma}(p)$ implies $\mathbf{b}_{\gamma}(q)=d(p, q)+\mathbf{b}_{\gamma}(p)=$ $d(p, q)+c-l=c$ and hence $q \in \mathbf{b}_{\gamma}^{-1}(c)$ and $q \in \sigma$, that is $q \in S_{c}$. These show that $S_{c}$ is non-empty and (i) is proved.

Proof of (ii). Next, we prove by contradiction that $S_{c}$ is open. Indeed, assuming by contradiction that for $q \in S_{c}$ there is a points sequence $\left\{q_{j}\right\} \subset \mathbf{b}_{\gamma}^{-1}(c) \backslash S_{c}$ such that $q=\lim _{j \rightarrow \infty} q_{j}$. We denote by $\sigma_{j}$ and $\sigma$ the co-rays passing through $q_{j}$ and $q$, respectively. Let $x$ be the initial point of $\sigma$, and by our assumption $x \in \mathcal{C}_{\gamma} \cap \mathbf{b}_{\gamma}^{-1}(-\infty, c]$.

Consider now a scalar $r>d(q, x)$ and the forward closed ball $\overline{\mathcal{B}^{+}(q, r)}:=\{p \in M \mid d(q, p) \leq r\}$. Obviously $\overline{\mathcal{B}^{+}(q, r)}$ is compact due to the forward complete hypothesis and the Hopf-Rinow theorem, and $x \in \overline{\mathcal{B}^{+}(q, r)}$.

Let $\sigma_{j}$ denote a co-ray to $\gamma$ emanating from $q_{j}=\sigma_{j}(0)$. Since $\overline{\mathcal{B}^{+}(q, r)}$ is compact and $q_{j} \notin S_{c}$, we can extend backward $\sigma_{j}$ to some interval $\left[s_{j}, 0\right]$ with $d\left(q, \sigma_{j}\left(s_{j}\right)\right)=r+\delta$. Any limit geodesic of the sequence $\left\{\sigma_{j}\right\}$ is a co-ray passing through $q$ which contains $x$ as an interior point, that is a contradiction (see Fig. 1).

It follows that $S_{c}$ must be open set and (ii) is proved.

Proof of (iii). Now we show that $\widetilde{M}$ is closed in $M$. Indeed let $\left\{x_{j}\right\}$ be a set of points in $\widetilde{M}$, such that $x_{j} \rightarrow x$ in $M$, and let $\sigma_{j}:\left(a_{j}, \infty\right) \rightarrow M$ be maximal co-rays to $\gamma$, parametrised such that $\sigma_{j}(0)=x_{j}$, with $a_{j} \in[-\infty, 0)$. Obviously, such $\sigma_{j}$ exist from the definition of $\widetilde{M}$. 


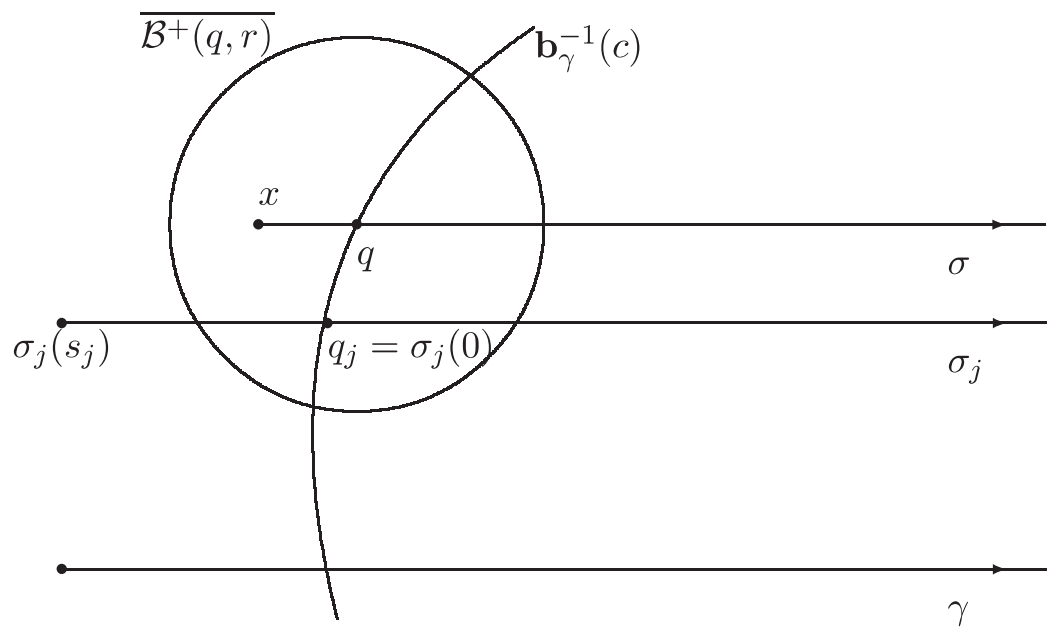

Figure 1. $S_{c}$ is an open set.

By extracting some sub-sequence of $\sigma_{j}$ we can assume, without loosing the generality, that $\left.\sigma_{j}\right|_{[0, \infty)}$ converges to some $\left.\sigma_{x}\right|_{[0, \infty)}$.

We will show that $x \in \widetilde{M}$. As the sequence $\left\{x_{j}\right\}$ was arbitrary, this would imply $\widetilde{M}$ closed. So assume by contradiction $x \in M \backslash \widetilde{M}$. This means that the domain of definition of the maximal co-ray extension of $\sigma_{x}$ is the interval $[a, \infty)$, for some $a \in[-\infty, 0)$. Therefore $\sigma_{x}(a) \in \mathcal{C}_{\gamma}$, and hence $q:=\sigma_{x}(1) \in S_{c}$, for $c:=1+\mathbf{b}_{\gamma}(x)$, where we use Theorem 1.1.

On the other hand, for $j$ large enough, consider the points $q_{j}:=\left.\sigma_{j}\right|_{[0, \infty)} \cap \mathbf{b}_{\gamma}^{-1}(c)$, and observe that $q_{j} \in \mathbf{b}_{\gamma}^{-1}(c) \backslash S_{c}$, by definition. But this contradicts the fact that $S_{c}$ is open, a fact proved already in (ii).

Thus, the (unique) maximal co-ray through $x$ must be of the form $\sigma_{x}:(a, \infty) \rightarrow M$, for some $a \in[-\infty, 0)$, and therefore $x \in \widetilde{M}$. This shows that $\widetilde{M}$ is closed and hence (iii) is proved.

Proof of (iv). Finally, we prove $\widetilde{M}$ is open set in $M$, or, equivalently, that $\widetilde{M} \backslash M$ is closed, in a similar manner.

Consider a sequence $\left\{x_{j}\right\}$ in $\widetilde{M} \backslash M$ with $x_{j} \rightarrow x$ in $M$, and consider the maximal co-rays $\sigma_{j}:\left[a_{j}, \infty\right) \rightarrow M$, with $\sigma_{j}(0)=x_{j}$. Obviously this is the form of the maximal co-rays due to the choice of $x_{j}$ and definition of $\widetilde{M}$.

Observe that the sequence of points $\left\{\sigma_{j}\left(a_{j}\right)\right\} \subset \mathcal{C}_{\gamma} \cap \mathbf{b}_{\gamma}^{-1}(-\infty, c]$, for $c:=1+\mathbf{b}_{\gamma}(1)$, and by the compactness hypothesis of $\mathcal{C}_{\gamma} \cap \mathbf{b}_{\gamma}^{-1}(-\infty, c]$ it follows that the limit point $\sigma_{x}(a) \in \mathcal{C} \cap \mathbf{b}_{\gamma}^{-1}(-\infty, c]$. Thus $x \in \widetilde{M} \backslash M$, and hence $\widetilde{M}$ is open, so (iv) is proved.

Using these we will build our argument as follows.

Reminding ourselves that a topological space $X$ is connected if and only if the only closed and open sets are the empty set and $X$, by taking $X=M$, and using claims (iii) and (iv) proved above, that is $\widetilde{M}$ is closed and open in $M$ it follows $\widetilde{M}=\varnothing$ or $\widetilde{M}=M$ (obviously $M$ is connected by hypothesis). However, since $S_{c} \neq \varnothing$ (claim (i) proved above), the maximal co-ray passing through any point $x \in S_{c}$ has the form $\sigma_{x}:[a, \infty) \rightarrow M$, so $\widetilde{M}=M$ cannot be possible, hence $\widetilde{M}=\varnothing$.

We obtain $S_{c}=\mathbf{b}_{\gamma}^{-1}(c)$. Indeed, $S_{c} \subset \mathbf{b}_{\gamma}^{-1}(c)$ by definition. Conversely, for any $q \in \mathbf{b}_{\gamma}^{-1}(c)$, it is now clear that there exists a maximal co-ray $\sigma_{q}:[a, \infty) \rightarrow M$ to $\gamma$ through $q$, hence $q \in S_{c}$ and the claim $S=\mathbf{b}_{\gamma}^{-1}(c)$ is proved.

We discuss now the case $c=\inf \mathbf{b}_{\gamma}(M)$. Firstly, we observe that, for any $c \geq \inf \mathbf{b}_{\gamma}(M)$, if $\sigma:(-\varepsilon, \infty) \rightarrow M$ is a co-ray such that $\sigma(0) \in \mathbf{b}_{\gamma}^{-1}(c)$, then $\mathbf{b}_{\gamma}\left(\sigma\left(-\frac{\varepsilon}{2}\right)\right)<c$, and thus $\mathbf{b}_{\gamma}^{-1}\left(\inf \mathbf{b}_{\gamma}(M)\right) \cap \widetilde{M}=\varnothing$. 
In particular, if $c=\inf \mathbf{b}_{\gamma}(M)$, and $\mathbf{b}_{\gamma}^{-1}(-\infty, c]=\mathbf{b}_{\gamma}^{-1}(c)$ is non-empty, then $S_{c}=\mathbf{b}_{\gamma}^{-1}(c)$. The proof of this fact is similar to the proof of (i).

Observe that this immediately implies that $S_{c} \neq \varnothing$ for all $c \geq \inf \mathbf{b}_{\gamma}(M)$.

In other words, what we have proved in Step 1 is that for any point $q \in \mathbf{b}_{\gamma}^{-1}(c)$, there exists a maximal co-ray, i.e., a co-ray emanating from a point $x \in \mathcal{C}_{\gamma}$, passing through $q$.

Step 2. Using this we proceed to proving that $\mathbf{b}_{\gamma}^{-1}(-\infty, c]$ is compact. We assume the converse, i.e., we assume there exists a divergent sequence $\left\{x_{j}\right\}$ in $\mathbf{b}_{\gamma}^{-1}(-\infty, c]$ in the sense that for any compact set $K \subset \mathbf{b}_{\gamma}^{-1}(-\infty, c]$, there exists $N_{K}>0$ such that $x_{j} \notin K$ for any $j>N_{K}$.

For each $j$ there exists a co-ray $\sigma_{j}$ from $x_{j}$ such that $\sigma_{j} \cap \mathbf{b}_{\gamma}^{-1}(c)=\left\{y_{j}\right\}$ (this can be easily seen by a similar argument as in the proof of $S \neq \varnothing$ ). From Step 1 it follows that we can extend $\sigma_{j}$ up to the point $z_{j}=\sigma_{j}(0) \in \mathcal{C}_{\gamma}$.

From hypothesis 2 of the theorem, there exists a subsequence $z_{j_{k}}$ of $z_{j}$ convergent to $z$ and hence there exists a sequence of co-rays $\sigma_{j_{k}}$ (emanating from each $z_{j_{k}}$ ) convergent to a co-ray $\sigma$ (emanating from the limit point $z$ ). For the sake of simplicity we assume $\lim _{j \rightarrow \infty} z_{j}=z$. It follows that there exists a point $y \in \mathbf{b}_{\gamma}^{-1}(c)$ such that $\lim _{j \rightarrow \infty} y_{j}=y$.

Since $x_{j}$ is by construction an interior point of the $N_{\gamma}^{c}$-segment $\left.\sigma_{j}\right|_{\left[0, s_{j}\right]}$ that joins $z_{j}$ to $y_{j}$, it follows that there exists a point $x$ interior to the $N_{\gamma}^{c}$-segment $\left.\sigma\right|_{[0, s]}$ that joins $z$ to $y$. But this implies that the sequence $\left\{x_{j}\right\}$ cannot be divergent in the sense above, that is we obtain a contradiction. Therefore, $\mathbf{b}_{\gamma}^{-1}(-\infty, c]$ must be compact.

\section{Remark 3.2.}

1. Observe that the conclusion of the theorem above cannot hold for $c<\inf \mathbf{b}_{\gamma}(M)$ since, in this case, $\mathbf{b}_{\gamma}^{-1}(-\infty, c]$ would be empty set.

2. Similarly with the proof of (ii) above, one can show that actually $S_{c}$ is also closed. We will not prove this property here because we don't need it.

Corollary 3.3. Let $(M, F)$ be a forward complete non-compact Finsler manifold and $\gamma$ a ray in $M$. If there exists a numerical sequence $\left\{c_{i}\right\}$ with $\lim _{i \rightarrow \infty} c_{i}=+\infty$, such that for each $i$ such that $\mathcal{C}_{\gamma} \cap \mathbf{b}_{\gamma}^{-1}\left(-\infty, c_{i}\right]$ is compact and non-empty, then set $\mathbf{b}_{\gamma}^{-1}\left(-\infty, c_{i}\right]$ is compact, i.e., the Busemann function $\mathbf{b}_{\gamma}$ is an exhaustion function.

The following lemma shows that Innami's result in [4] is a special case of our Theorem 3.1.

Lemma 3.4. Let $(M, F)$ be a bi-complete Finsler manifold and $\gamma$ a ray in $M$. If $\mathcal{C}_{\gamma} \neq \varnothing$ is compact, then for all sufficiently large $a \in \mathbb{R}$, the level set $\mathbf{b}_{\gamma}^{-1}(a)$ is arcwise connected.

Proof. Since $\mathcal{C}_{\gamma} \neq \varnothing$ is compact we can choose a number $a>\max \mathbf{b}_{\gamma}\left(\mathcal{C}_{\gamma}\right)$. Thus there does not exist a co-point of $\gamma$ in $\mathbf{b}_{\gamma}^{-1}[a, \infty)$. Choose any two points $x$ and $y$ in $\mathbf{b}_{\gamma}^{-1}(a)$. By Lemma 2.3, there exists a continuous curve $c$ in $\mathbf{b}_{\gamma}^{-1}[a, \infty)$ joining $x$ to $y$. Since $\mathcal{C}_{\gamma} \cap \mathbf{b}_{\gamma}^{-1}[a, \infty)=\varnothing$, we can get a curve in $\mathbf{b}_{\gamma}^{-1}(a)$ joining $x$ to $y$ by deforming the curve $c$ along the co-rays intersecting $c$. Therefore, the level set is arcwise connected.

Remark 3.5. Observe that the bi-completeness hypothesis is needed for deforming the curve $c$ above.

Moreover, we have

Theorem 3.6. Let $(M, F)$ be a forward complete non-compact boundaryless Finsler manifold and $\gamma$ a ray in $M$. If $\mathcal{C}_{\gamma}$ contains an isolated point $p$, then

1. The exponential map $\exp _{p}: T_{p} M \rightarrow M$ is a $C^{1}$-diffeomorphism and any geodesic emanating from $p$ is a maximal co-ray to $\gamma$. 
2. $\mathcal{C}_{\gamma}=\{p\}$ only.

3. For any fixed point $q \in M$, the relation

$$
d(p, q)+\mathbf{b}_{\gamma}(p)=\mathbf{b}_{\gamma}(q)
$$

holds good. In particular, for any $a>\mathbf{b}_{\gamma}(p)$ the level sets $\mathbf{b}_{\gamma}^{-1}(a)$ coincide with the forward spheres $\mathcal{S}^{+}\left(p, a-\mathbf{b}_{\gamma}(p)\right):=\left\{q \in M: d(p, q)=a-\mathbf{b}_{\gamma}(p)\right\}$.

Proof. 1. Since $p \in \mathcal{C}_{\gamma}$ is isolated in $\mathcal{C}_{\gamma}$, it follows by definition that there exists $\varepsilon_{0}>0$ such that

$$
\mathcal{C}_{\gamma} \cap \mathcal{B}^{+}\left(p, \varepsilon_{0}\right)=\{p\},
$$

where $\mathcal{B}^{+}\left(p, \varepsilon_{0}\right)$ is the forward ball in $(M, F)$.

Claim 1. There exists $\varepsilon_{1} \in\left(0, \varepsilon_{0}\right)$ such that any co-ray to $\gamma$ emanating from a point of $M \backslash \mathcal{B}^{+}\left(p, \varepsilon_{0}\right)$ does not intersect $\mathcal{B}^{+}\left(p, \varepsilon_{1}\right)$.

Indeed, let us assume the contrary, that is, we shall assume that for each positive integer $j \in$ $\{1,2, \ldots\}$, there exists a co-ray $\sigma_{j}:[0, \infty) \rightarrow M$, emanating from a point $q_{j}=\sigma_{j}(0) \notin \mathcal{B}^{+}\left(p, \varepsilon_{0}\right)$, that intersects $\mathcal{B}^{+}\left(p, \frac{1}{j}\right)$.

Under this assumption, by extracting a subsequence of $\left\{\sigma_{j}\right\}$ we can construct a convergent sequence of co-rays with the properties in the assumption above. For simplicity, we denote this subsequence by $\left\{\sigma_{j}\right\}$ again. In this way, we obtain a limit co-ray $\sigma:=\lim _{j \rightarrow \infty} \sigma_{j}$, and a convergent sequence of points $\left.p_{j} \in \mathcal{B}^{+}\left(p, \frac{1}{j}\right) \cap \sigma_{j}\right|_{[0, \infty)}, \lim _{j \rightarrow \infty} p_{j}=p$. It follows that there exists a co-ray $\sigma$ and $p$ is interior point of $\sigma$. This is a contradiction with $p \in \mathcal{C}_{\gamma}$ and Claim 1 is proved.

Claim 2. For any point $q \in \mathcal{B}^{+}\left(p, \varepsilon_{1}\right)$, the geodesic emanating from $p$ and passing through the point $q$ is a co-ray of $\gamma$.

Let $\sigma:[0, \infty) \rightarrow M$ be a co-ray to $\gamma$ emanating from $q=\sigma(0)$, and let $\widetilde{\sigma}:(a, \infty) \rightarrow M$ be the maximal geodesic extension of $\sigma$. One of the following situations happen.

Case 1. $\left.\tilde{\sigma}\right|_{(a, 0]} \subset \mathcal{B}^{+}\left(p, \varepsilon_{0}\right)$.

In this case, since $\mathcal{B}^{+}\left(p, \varepsilon_{0}\right)$ is compact, there exists $b \in(a, 0)$ such that $\left.\widetilde{\sigma}\right|_{[b, 0]}$ is not minimal. In particular, $\left.\widetilde{\sigma}\right|_{[b, \infty)}$ is not a ray.

Thus, there must exist $b_{1} \in(b, 0]$ such that $\left.\widetilde{\sigma}\right|_{\left[b_{1}, \infty\right)}$ is a maximal co-ray to $\gamma$. It follows $\widetilde{\sigma}\left(b_{1}\right) \in \mathcal{C}_{\gamma} \cap \mathcal{B}^{+}\left(p, \varepsilon_{0}\right)=\{p\}$ and hence $\left.\widetilde{\sigma}\right|_{\left[b_{1}, \infty\right)}$ is a co-ray to $\gamma$ emanating from $p$ and passing through the point $q$.

Case 2. There exists $b \in(a, 0]$ such that $\widetilde{\sigma}(b) \notin \mathcal{B}^{+}\left(p, \varepsilon_{0}\right)$.

In this case, let us denote $b_{1}:=\max \left\{t<0: d(p, \widetilde{\sigma}(t))=\varepsilon_{0}\right\}$. Since $\widetilde{\sigma}(0)=q \in \mathcal{B}^{+}\left(p, \varepsilon_{1}\right)$, it results that $\left.\widetilde{\sigma}\right|_{\left[b_{1}, \infty\right)}$ is not a co-ray to $\gamma$ and $\left.\widetilde{\sigma}\right|_{\left(b_{1}, 0\right)} \subset \mathcal{B}^{+}\left(p, \varepsilon_{0}\right)$.

Therefore, it must exist $b_{2} \in\left(b_{1}, 0\right)$ such that $\left.\widetilde{\sigma}\right|_{\left[b_{2}, \infty\right)}$ is a maximal co-ray to $\gamma$ passing through $\widetilde{\sigma}\left(b_{2}\right) \in \mathcal{C}_{\gamma} \cap \mathcal{B}^{+}\left(p, \varepsilon_{0}\right)=\{p\}$.

From these it results that for any point $q \in \mathcal{B}^{+}\left(p, \varepsilon_{1}\right)$, there exists a co-ray to $\gamma$ emanating from $p$ and passing through the point $q$ and Claim 2 is proved.

From Claims 1 and 2 we conclude that any geodesic from $p$ is a co-ray to $\gamma$.

It follows now from Theorem 1.2 that any two distinct co-rays of $\gamma$ emanating from $p$ do not intersect again. Indeed, it is trivial to see that since $\mathbf{b}_{\gamma}$ is differentiable at interior points of co-rays and the tangent direction of the co-ray at such a point is $\nabla \mathbf{b}_{\gamma}$, it is not possible for co-rays to intersect each other at their interior points.

In this way we obtain that $\exp _{p}: T_{p} M \rightarrow M$ is a $C^{1}$-diffeomorphism and first statement of the theorem is proved.

2. The fact that $C_{\gamma}=\{p\}$ it is now obvious from the proof of 1 .

3. Let us choose any point $q \in M \backslash\{p\}$, and let us denote by $\beta:[0, \infty) \rightarrow M$ any unit speed geodesic emanating from $p$ and passing through the point $q$. From the first statement of this 
theorem it follows that $\beta$ must be a co-ray to $\gamma$ and hence the relation $\mathbf{b}_{\gamma}(\beta(s))=s+\mathbf{b}_{\gamma}(p)$ holds for any $s \geq 0$. In particular, since $q=\beta(d(p, q))$, it results $\mathbf{b}_{\gamma}(q)=d(p, q)+\mathbf{b}_{\gamma}(p)$.

Moreover, from here it follows that for any $a>\mathbf{b}_{\gamma}(p)$, we have $\mathbf{b}_{\gamma}^{-1}(a)=\mathcal{S}^{+}\left(p, a-\mathbf{b}_{\gamma}(a)\right)$, and the theorem is proved.

Remark 3.7. If $(M, F)$ is a non-compact Finsler manifold whose all geodesics are strightlines, then $(M, F)$ is bi-complete and $\mathcal{C}_{\gamma}=\varnothing$.

Remark 3.8. It would be interesting to obtain some geometrical conditions (flag curvature conditions) on the Finsler manifold $(M, F)$ such that all Busemann functions are everywhere differentiable. Since this topic requires more elaboration, we leave it for a future research.

We recall that an end $\varepsilon$ of a non-compact manifold $X$ is an assignment to each compact set $K \subset X$ a component $\varepsilon(K)$ of $X \backslash K$ such that $\varepsilon\left(K_{1}\right) \supset \varepsilon\left(K_{2}\right)$ if $K_{1} \subset K_{2}$. Every non-compact manifold has at least one end. For instance, $\mathbb{R}^{n}$ has one end if $n>1$ and two ends if $n=1$. By definition one can see that a product $\mathbb{R} \times N$ has one end if $N$ is non-compact and two ends otherwise.

Here we prove

Corollary 3.9. Let $(M, F)$ be a bi-complete non-compact Finsler manifold.

1. If $\mathcal{C}_{\gamma}=\varnothing$, then $M$ is homeomorphic to $\mathbb{R} \times \mathbf{b}_{\gamma}^{-1}(0)$.

2. If $M$ has at least three ends, then there are no differentiable Busemann functions on $M$.

Proof. 1. Since $\mathcal{C}_{\gamma}=\varnothing$, it follows that $\mathbf{b}_{\gamma}$ is smooth everywhere and hence from each point there is a unique co-ray to $\gamma$. Thus, we can define the function $\varphi: M \rightarrow \mathbb{R} \times \mathbf{b}_{\gamma}^{-1}(0), p \mapsto$ $\varphi\left(\mathbf{b}_{\gamma}(p), h_{1}(p)\right)$, where $h_{1}(p)$ is the intersection point of the co-ray from $p$ with the level set $\mathbf{b}_{\gamma}^{-1}(0)$. From the bi-completness hypothesis it follows that $h_{1}(p)$ always exists. We can easily see that this is a homeomorphism by using the Lipschitz continuity of $\mathbf{b}_{\gamma}$.

2. Due to statement 1 it follows that if $\mathbf{b}_{\gamma}$ is differentiable, then $M$ have at most two ends. Statement 2 follows by logical negation.

Remark 3.10. It is known that the cut locus of a point in a Riemannian or Finsler manifold $M$ is a closed subset of $M$ (see [1]). On the other hand, we have shown in [12], by an example, that the cut locus of a closed subset in $M$ is not closed in $M$ anymore. A natural question is if the co-point set $\mathcal{C}_{\gamma}$ is closed or not. First to answer to this question is Nasu who constructed in [7] an example of Riemannian complete surface with a ray $\gamma$ whose co-point set $\mathcal{C}_{\gamma}$ is not closed. Obviously, the same conclusion can be derived from our Theorem 1.2 and [12].

\section{Acknowledgements}

I am grateful to Professor M. Tanaka for bringing this topic into my attention and for many illuminating discussions. I am also deeply indebted to the anonymous referees for their constructive criticism and extremely useful suggestions that improved the manuscript enormously. Also I thank to N. Boonnam for reading an early version of the paper.

\section{References}

[1] Bao D., Chern S.-S., Shen Z., An introduction to Riemann-Finsler geometry, Graduate Texts in Mathematics, Vol. 200, Springer-Verlag, New York, 2000.

[2] Busemann H., The geometry of geodesics, Academic Press Inc., New York, N.Y., 1955.

[3] Egloff D., Uniform Finsler Hadamard manifolds, Ann. Inst. H. Poincaré Phys. Théor. 66 (1997), 323-357. 
[4] Innami N., Differentiability of Busemann functions and total excess, Math. Z. 180 (1982), 235-247.

[5] Innami N., On the terminal points of co-rays and rays, Arch. Math. (Basel) 45 (1985), 468-470.

[6] Lewis G.M., Cut loci of points at infinity, Pacific J. Math. 43 (1972), 675-690.

[7] Nasu Y., On asymptotes in a metric space with non-positive curvature, Tôhoku Math. J. 9 (1957), 68-95.

[8] Ohta S.-I., Splitting theorems for Finsler manifolds of nonnegative Ricci curvature, J. Reine Angew. Math. 700 (2015), 155-174.

[9] Shen Z., Lectures on Finsler geometry, World Scientific Publishing Co., Singapore, 2001.

[10] Shiohama K., Topology of complete noncompact manifolds, in Geometry of Geodesics and Related Topics (Tokyo, 1982), Adv. Stud. Pure Math., Vol. 3, North-Holland, Amsterdam, 1984, 423-450.

[11] Shiohama K., Shioya T., Tanaka M., The geometry of total curvature on complete open surfaces, Cambridge Tracts in Mathematics, Vol. 159, Cambridge University Press, Cambridge, 2003.

[12] Tanaka M., Sabau S.V., The cut locus and distance function from a closed subset of a Finsler manifold, Houston J. Math., to appear, arXiv:1207.0918. 